\title{
Efficacy of bendamustine on thrombocytopenia and hemolytic anemia secondary to CD5-positive B-cell lymphoma with massive splenomegaly in a patient with rheumatoid arthritis
}

\author{
YUZURU HOSODA ${ }^{1,2}$, HIROSHI HAGINO ${ }^{3}$, NORIHIKO HINO ${ }^{2}$ and TORU MOTOKURA ${ }^{1,2}$ \\ ${ }^{1}$ Division of Clinical Laboratory Medicine, Department of Pathophysiological and Therapeutic Science, \\ Tottori University Faculty of Medicine, Yonago, Tottori 683-8503; ${ }^{2}$ Department of Hematology and \\ ${ }^{3}$ Division of Rehabilitation, Tottori University Hospital, Yonago, Tottori 683-8504, Japan
}

Received June 14, 2017; Accepted September 13, 2017

DOI: $10.3892 / \mathrm{mco} .2017 .1425$

\begin{abstract}
Chemotherapy for lymphoma may be avoided in the presence of coincident cytopenia. In case of immune cytopenia secondary to lymphoma, treatment of cytopenia is the same for primary cases, however, chemotherapy for lymphoma may be effective at the cost of severe hematological toxicity. The present study reports a complex case of thrombocytopenia and direct antiglobulin test-negative hemolytic anemia, thus mimicking Evans syndrome, secondary to cluster of differentiation 5-positive B-cell lymphoma with massive splenomegaly, in a patient suffering from rheumatoid arthritis for two decades. Treatment with prednisolone, high-dose dexamethasone, eltrombopag and rituximab for cytopenia were not effective. Chemotherapy with bendamustine subsequently resolved the cytopenia, additionally resulting in a complete remission of lymphoma. Thus, bendamustine may have a role in the management of lymphoma complicated with severe cytopenia.
\end{abstract}

\section{Introduction}

Autoimmune diseases including rheumatoid arthritis are associated with a higher risk of non-Hodgkin lymphoma (1) and the frequency of autoimmune disease in lymphoma patients was $8.2 \%$ (2). Among autoimmune diseases, combination of immune thrombocytopenia and autoimmune hemolytic

Correspondence to: Dr Toru Motokura, Division of Clinical Laboratory Medicine, Department of Pathophysiological and Therapeutic Science, Tottori University Faculty of Medicine, 86 Nishi-cho, Yonago, Tottori 683-8503, Japan

E-mail: motokura@med.tottori-u.ac.jp

Abbreviations: ITP, immune thrombocytopenia; DAT, direct antiglobulin test; LDH, lactic dehydrogenase; FDG, ${ }^{18}$ F-fluorodeoxyglucose; PET, positron emission tomography; CT, computed tomography

Key words: bendamustine, Evans syndrome, immune thrombocytopenia, splenomegaly, rheumatoid arthritis anemia, which is known as Evans syndrome, may precede lymphoma $(3,4)$. Treatment of these cytopenias is the same for primary cases although coincident presence of lymphoma is a clinical problem. Conventional chemotherapy of lymphoma may be avoided in the presence of severe cytopenia because of the increased risk of hematological toxicity, however, treatment of lymphoma may be needed for the few patients with lymphoma-associated cytopenia.

In the present study, we report a complex case of thrombocytopenia and hemolytic anemia secondary to CD5-positive B-cell lymphoma in a patient with massive splenomegaly and rheumatoid arthritis, which responded to chemotherapy with bendamustine.

\section{Case report}

The present study focused on a 67-year-old Japanese woman who developed splenomegaly and mild neutropenia after a 20 -year course of rheumatoid arthritis which had been in remission with tofacitinib treatment. In addition, she developed thrombocytopenia and anemia and tofacitinib was terminated. Laboratory examination revealed the followings: Reticulocyte $248 \times 10^{9} / 1$, total bilirubin $1.9 \mathrm{mg} / \mathrm{dl}$, lactic dehydrogenase (LDH) $438 \mathrm{U} / 1$ (upper normal limit $223 \mathrm{U} / \mathrm{l}$ ), direct anti-globulin test (DAT) negative and platelet-associated immunoglobulin G $123 \mathrm{ng} / 10^{7}$ cells (upper normal limit 46). Peripheral blood smear rarely showed fragmented red cells and there was no coagulopathy. Bone marrow aspirate and needle biopsy showed erythroid hyperplasia and no definite lymphoma infiltrates but several reactive lymphocyte aggregates. Tentative diagnosis of immune thrombocytopenia (ITP) and DAT-negative hemolytic anemia, thus DAT-negative Evans syndrome, was made in spite of massive splenomegaly. Prednisolone $30 \mathrm{mg} /$ day was administered to treat bicytopenia while abnormal lymphocytes appeared in peripheral blood (Fig. 1). Flow cytometric analysis showed the presence of monoclonal B-cells expressing CD5, CD19, CD20, HLA-DR, and surface IgM- $\lambda$ and partially positive for CD10 without CD23 expression. In addition, fluorescence in situ hybridization analysis of peripheral blood revealed an increased number of $M Y C$ and $I G H$ signals without any fusion in $40 \%$ of mononuclear cells examined, corroborating 


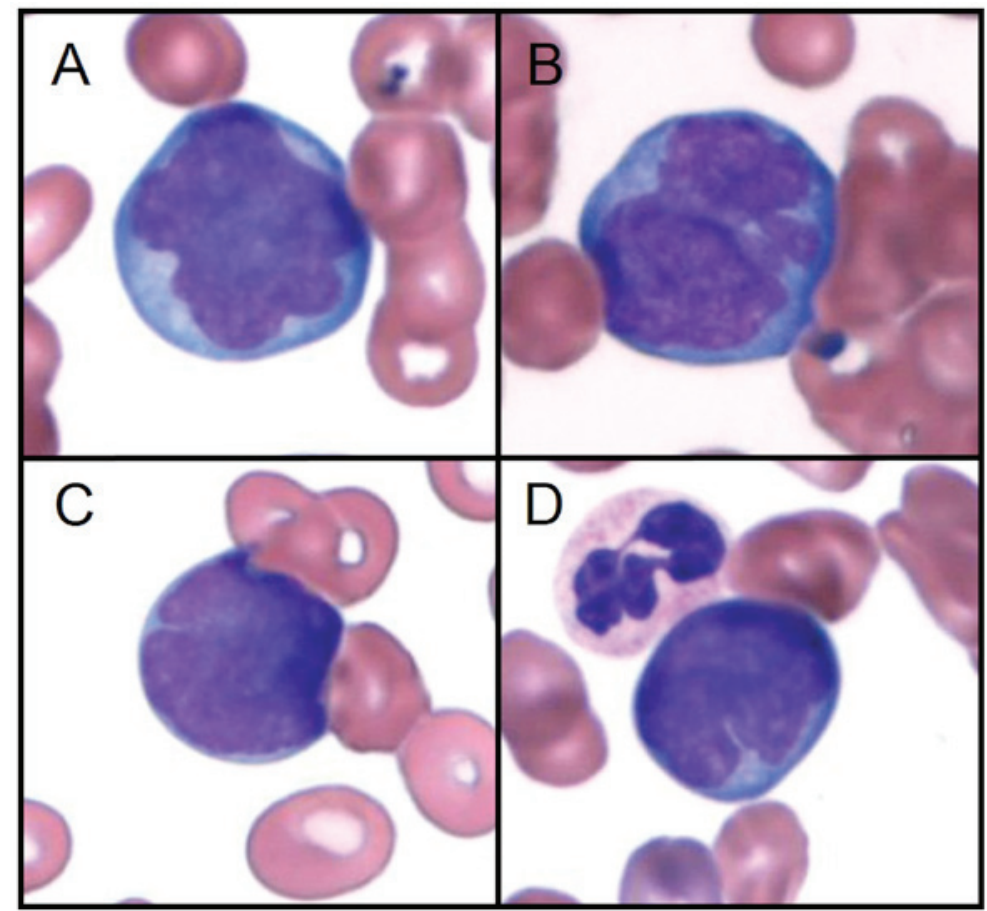

Figure 1. Abnormal lymphoid cells in the peripheral blood. (A-D) These cells were positive for CD5, CD19, CD20, HLA-DR, and surface IgM- $\lambda$, partially positive for CD10, and negative for CD23 except for a neutrophil in D.

A

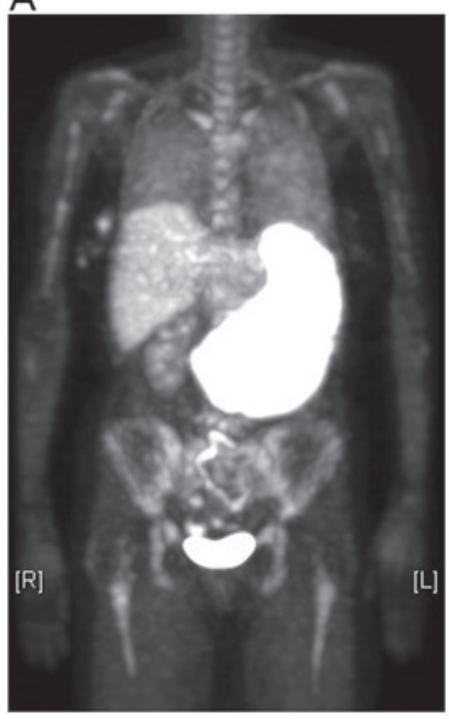

B

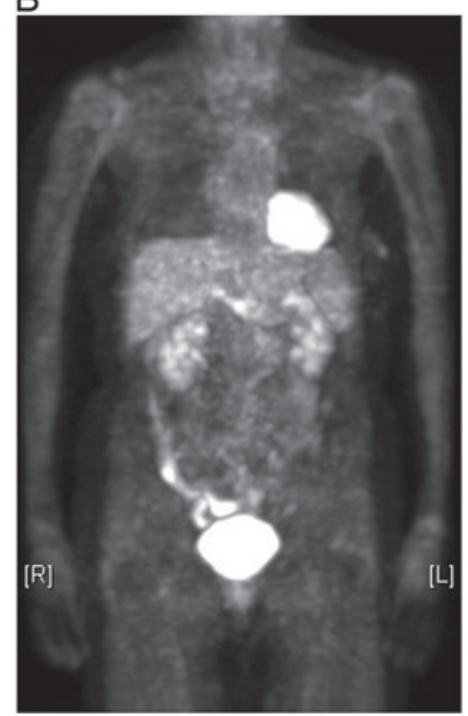

Figure 2. PET-CT scans. (A) Massive splenomegaly and hepatomegaly with high FDG uptakes in addition to diffuse FDG uptake in both lungs and bone marrow were found before chemotherapy. (B) Hepatosplenomegaly and FDG uptakes disappeared after chemotherapy.

the result of bone marrow cytogenetics which revealed later that 2 out of 20 cells were near-tetraploid cells with complex cytogenetics. Diagnosis of CD5-positive B-cell lymphoma was made. ${ }^{18} \mathrm{~F}$-fluorodeoxyglucose (FDG) positron emission tomography (PET)-computed tomography (CT) showed massive splenomegaly with high uptake of FDG as well as diffuse FDG uptake in both lungs and the bone marrow without any abnormal lung shadow in CT (Fig. 2A), suggesting intravascular large B-cell lymphoma; hypoxemia developed with peripheral oxygen saturation $88 \%$ (room air). Severe thrombocytopenia (platelet count $<10 \times 10^{9} / 1$ ) refractory to platelet transfusion developed and hindered diagnostic splenectomy or splenic biopsy for histological determination of lymphoma. We administered high-dose dexamethasone, high-dose $\gamma$-globulin and rituximab along with daily platelet transfusions and semi-weekly red blood cell transfusions to treat both severe bicytopenia and lymphoma. A transient decrease in number of lymphoma cells in the peripheral blood was observed after each dose of rituximab, however, no improvement of bicytopenia occurred. We administered second high-dose dexamethasone followed by prednisolone maintenance and start of eltrombopag $12.5 \mathrm{mg} /$ day, which was planned to be escalated weekly by 


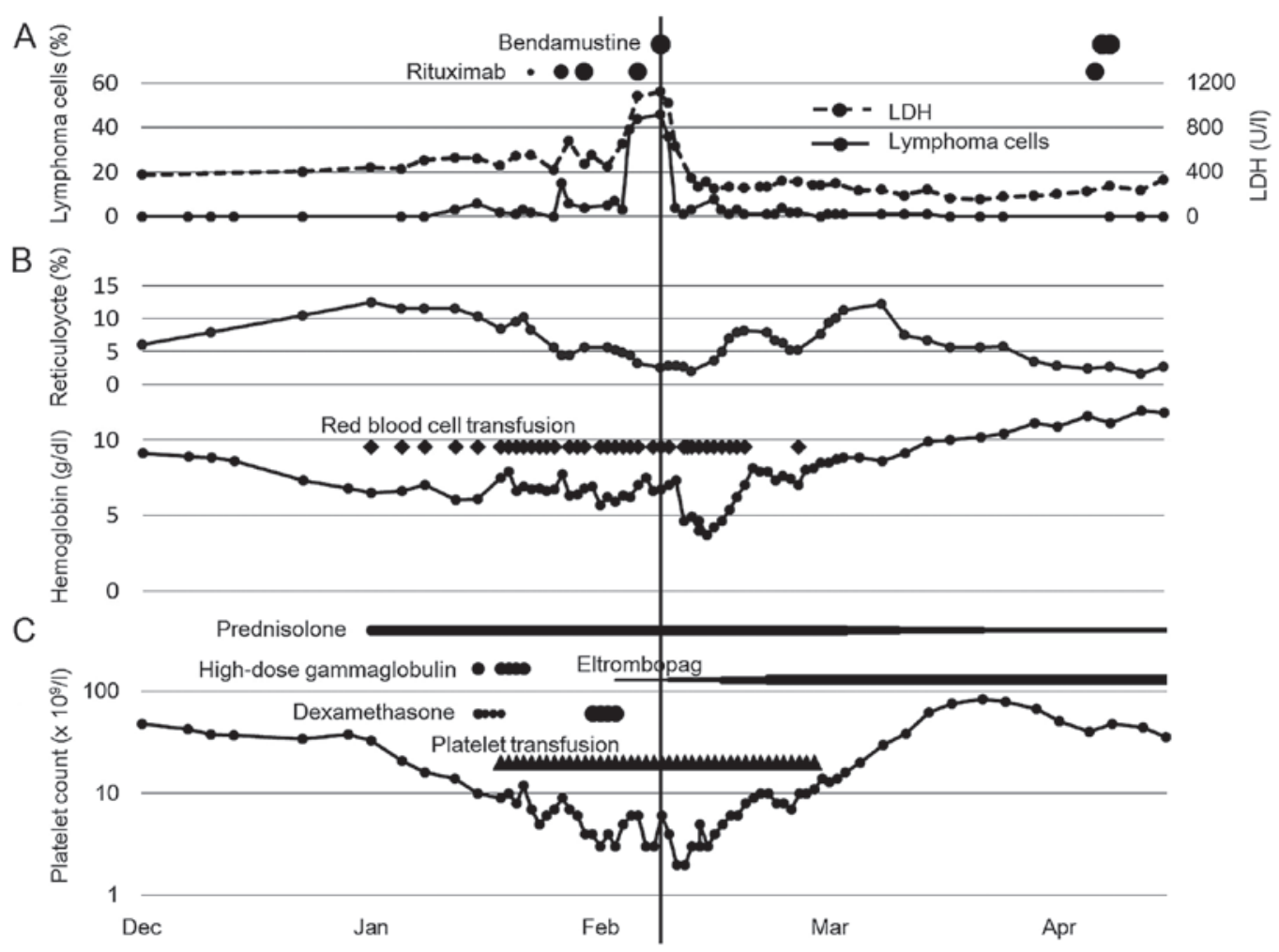

Figure 3. Time courses of (A) CD5-positive lymphoma treated with bendamustine and rituximab, (B) hemolytic anemia and (C) thrombocytopenia (A) Peripheral blood lymphoma cell numbers (\%) and serum LDH levels (U/1) are shown over time and correlated well. (B) Reticulocyte counts (\%) and hemoglobin levels $(\mathrm{g} / \mathrm{dl})$ are shown over time. (C) Platelet counts are shown as a logarithmic scale. The size of each circle and thickness of each line reflect the relative doses of the indicated drugs. The first dose of rituximab was split in two because of splenomegaly. A vertical line in the middle indicates the time of the initial bendamustine administration, which was given only one day because of severe jaundice. Each red blood cell transfusion, derived from $400 \mathrm{ml}$ blood, is shown as a diamond and each platelet transfusion, derived from 2,000 $\mathrm{ml}$ blood, as a triangle.

$12.5 \mathrm{mg}$ per day up to $50 \mathrm{mg}$ per day. Even after 3 doses of rituximab, the number of peripheral blood lymphoma cells increased as well as serum levels of LDH as shown in Fig. 3A. Then, we administered bendamustine $90 \mathrm{mg} / \mathrm{m}^{2}$ once. Due to high serum levels of total bilirubin $(8.8 \mathrm{mg} / \mathrm{dl})$, second dose of bendamustine was omitted. Severe pancytopenia developed; leukocytes $1.9 \times 10^{9} / 1$, hemoglobin $3.7 \mathrm{~g} / \mathrm{dl}$, and platelets $3 \times 10^{9} / 1$ at nadir of bone marrow suppression (Fig. 3B and C). Three weeks later, transfusion-independence for both of erythrocytes and platelets was obtained while cytomegalovirus antigenemia had become positive and severe pneumonia had developed. Antiviral, antibiotic and antifungal treatments were administered successfully to overcome these complications. Spleen decreased in size and pancytopenia ultimately disappeared as well as lymphoma cells in the peripheral blood. She received additional 5 courses of bendamustine plus rituximab therapy in full doses (5) and PET-CT scans revealed complete disappearance of FDG uptakes and splenomegaly (Fig. 2B).

The patient has provided her consent regarding the publication of the case details. The present study was undertaken according to a project of clinical surveillance in Tottori University Hospital, which was approved by the Ethics Committee at Tottori University Faculty of Medicine (approval no. 2489).

\section{Discussion}

Massive splenomegaly may constitute the initial presentation of lymphoma. In the subset of patients in which splenomegaly remains unexplained, lymphomas account for the leading cause and splenectomy is required for a definitive diagnosis although primary effort must be taken to achieve diagnosis by integrating presenting clinical and laboratory data with imaging, peripheral blood, bone marrow assessment, thus trying to avoid splenectomy for diagnostic purposes (6). In the current case, bone marrow examination gave no definitive diagnosis and severe thrombocytopenia hindered diagnostic splenectomy while CD5-positive monoclonal B cells with bizarre morphology and complex cytogenetics appeared in the peripheral blood. Taken splenomegaly and diffuse pulmonary involvement as suggested by the PET-CT scan into consideration, pulmonary intravascular large B-cell lymphoma is probable diagnosis $(7,8)$.

Evans syndrome is a rare disease characterized by the simultaneous and sequential development of autoimmune hemolytic anemia and ITP and/or immune neutropenia. Michel et al analyzed the data from 68 patients with Evans syndrome including 3 DAT-negative cases (4). Half of the patients were associated with an underlying disorder including lymphoproliferative disorders and two patients with B-cell non-Hodgkin lymphoma was included (4). The presence of massive splenomegaly suggested against autoimmune mechanisms underlying bicytopenia in the current case although the severity of bicytopenia refractory to multiple transfusions could not negate the presence of immune dysregulation like Evans syndrome especially because the patient suffered from rheumatoid arthritis. Whether it was via immunological 
mechanisms or not, severe bicytopenia was a clinical problem confronting lymphoma progression. Park et al reported a case of DAT-negative Evans syndrome associated with diffuse large B-cell lymphoma, in which cytopenia preceded lymphoma and R-CHOP chemotherapy was effective (3). Multirefractory ITP is a serious and potentially life-threatening condition although chemotherapy may be needed for the few patients with ITP secondary to lymphoma (9). In the current case, prednisolone, high-dose $\gamma$-globulin, high-dose dexamethasone, rituximab and eltrombopag failed to increase platelet counts and hemoglobin levels. In addition, peripheral lymphoma cells increased in number and serum levels of LDH elevated despite repeated rituximab doses. Then, administration of bendamustine successfully eradicated lymphoma cells from the peripheral blood and increased platelet counts. Furthermore, anemia, although transiently aggravated, ultimately disappeared. Bendamustine is effective for indolent and mantle cell lymphoma (5) and even aggressive lymphoma $(10,11)$. Bendamustine plus rituximab was better tolerated than R-CHOP with lower rates of myelotoxicity while higher frequency of grade 3-4 lymphocytopenia was seen in the bendamustine plus rituximab group (5). Bendamustine may be favored for patients with lymphoma associated with severe cytopenia because of less myelotoxicity. Furthermore, lymphotoxicity of bendamustine may affect autoimmune cytopenia favorably.

In conclusion, we here report a complex case of severe bicytopenia and B-cell lymphoma in a patient with rheumatoid arthritis. Bendamustine was successful for both diseases and may be preferred in case of lymphoma complicated with cytopenia.

\section{Acknowledgements}

The present study was supported in part by grants from Teijin Pharma Ltd., Chugai pharmaceutical Co., Ltd. and Taiho Pharmaceutical Co., Ltd.

\section{References}

1. Fallah M, Liu X, Ji J, Försti A, Sundquist K and Hemminki K: Autoimmune diseases associated with non-Hodgkin lymphoma: A nationwide cohort study. Ann Oncol 25: 2025-2030, 2014.
2. Váróczy L, Gergely L, Zeher M, Szegedi G and Illés A: Malignant lymphoma-associated autoimmune diseases-a descriptive epidemiological study. Rheumatol Int 22: 233-237, 2002.

3. Park SY, Kim S, Kim ES, Choi SU, Hyun HJ, Ahn JY, Lee JH, Ryu SH, Park JH, Lee GI and Lee HJ: A case of Non-Hodgkin's lymphoma in patient with coombs' negative hemolytic anemia and idiopathic thrombocytopenic purpura. Cancer Res Treat 44: 69-72, 2012.

4. Michel M, Chanet V, Dechartres A, Morin AS, Piette JC, Cirasino L, Emilia G, Zaja F, Ruggeri M, Andrès E, et al: The spectrum of Evans syndrome in adults: New insight into the disease based on the analysis of 68 cases. Blood 114: 3167-3172, 2009.

5. Rummel MJ, Niederle N, Maschmeyer G, Banat GA, von Grünhagen U, Losem C, Kofahl-Krause D, Heil G, Welslau M, Balser C, et al: Bendamustine plus rituximab versus CHOP plus rituximab as first-line treatment for patients with indolent and mantle-cell lymphomas: An open-label, multicentre, randomised, phase 3 non-inferiority trial. Lancet 381: 1203-1210, 2013.

6. Iannitto E and Tripodo C: How I diagnose and treat splenic lymphomas. Blood 117: 2585-2595, 2011.

7. Nishii-Ito S, Izumi H, Touge H, Takeda K, Hosoda Y, Yamasaki A, Kuwamoto S, Shimizu E and Motokura T: Pulmonary intravascular large B-cell lymphoma successfully treated with rituximab, cyclophosphamide, vincristine, doxorubicin and prednisolone immunochemotherapy: Report of a patient surviving for over 1 year. Mol Clin Oncol 5: 689-692, 2016.

8. Yousem SA and Colby TV: Intravascular lymphomatosis presenting in the lung. Cancer 65: 349-353, 1990.

9. Mahevas M, Gerfaud-Valentin M, Moulis G, Terriou L, Audia S, Guenin S, Le Guenno G, Salles G, Lambotte O, Limal N, et al: Characteristics, outcome, and response to therapy of multirefractory chronic immune thrombocytopenia. Blood 128: 1625-1630, 2016

10. Hitz F, Zucca E, Pabst T, Fischer N, Cairoli A, Samaras P, Caspar CB, Mach N, Krasniqi F, Schmidt A, et al: Rituximab, bendamustine and lenalidomide in patients with aggressive B-cell lymphoma not eligible for anthracycline-based therapy or intensive salvage chemotherapy-SAKK 38/08. Br J Haematol 174: 255-263, 2016.

11. Ohmachi K, Niitsu N, Uchida T, Kim SJ, Ando K, Takahashi N, Takahashi N, Uike N, Eom HS, Chae YS, et al: Multicenter phase II study of bendamustine plus rituximab in patients with relapsed or refractory diffuse large B-cell lymphoma. J Clin Oncol 31: 2103-2109, 2013. 